\title{
Inhomogeneous temperature dependence of the magnetization in fcc-Fe on $\mathrm{Cu}(001)$
}

\author{
C. E. ViolBarbosa,${ }^{1}$ H. L. Meyerheim,,${ }^{1}{ }^{*}$ E. Jal,${ }^{2}$ J.-M. Tonnerre ${ }^{2}$ M. Przybylski, ${ }^{1}$ L. M. Sandratskii, ${ }^{1}$ F. Yildiz, ${ }^{1}$ \\ U. Staub, ${ }^{3}$ and J. Kirschner ${ }^{1}$ \\ ${ }^{1}$ Max-Planck-Institut für Mikrostrukturphysik, Weinberg 2, D-06120 Halle, Germany \\ ${ }^{2}$ Institut Néel, CNRS et Universitè J. Fourier, F-38043 Grenoble, France \\ ${ }^{3}$ Swiss Light Source, Paul Scherrer-Institut, CH-5232 Villigen, Switzerland \\ (Received 6 January 2012; revised manuscript received 19 April 2012; published 14 May 2012)
}

\begin{abstract}
Using soft-x-ray magnetic resonant reflectivity measurements, we have studied the layer-resolved temperature dependence of the magnetic structure in a six-monolayer-thick fcc-Fe film grown on $\mathrm{Cu}(001)$ by pulsed laser deposition. Temperature-dependent reflectivity curves were fitted on the basis of the Heisenberg Hamiltonian involving only a small number of temperature-independent interlayer and intralayer exchange interaction parameters. Our study supports the model of the formation of the blocks of layers with robust magnetic structure whereas the interblock interactions are relatively weak. The temperature-dependent magnetization profile derived on the basis of the exchange interaction parameters reveals a strong inhomogeneity within the film, which is in agreement with the recent theoretical prediction.
\end{abstract}

DOI: 10.1103/PhysRevB.85.184414

PACS number(s): 75.70.Cn, 61.05.cm, 75.70.Rf

\section{INTRODUCTION}

Ultrathin $\mathrm{Fe}$ films on $\mathrm{Cu}(001)$ have been investigated for more than two decades, motivated by the possibility of stabilizing $\mathrm{Fe}$ in the face-centered-cubic (fcc) lattice at room temperature (RT). The fcc phase of Fe is notorious for its magnetic properties, which are strongly dependent on subtle structural changes. ${ }^{1,2}$ Experimental studies demonstrate that Fe films grown on $\mathrm{Cu}(100)$ are ferromagnetic below four monolayers (MLs) of thickness, while the spin structure of thicker films in the 6-8 ML regime is a matter of ongoing discussion.

There is a wide consensus that the top two layers are ferromagnetically (FM) coupled, while the deeper layers are characterized by a nonferromagnetic ordering. ${ }^{3}$ The suggestions for the nonferromagnetic part of the film vary widely and include, among others, single-layer ${ }^{4,5}$ and double-layer collinear antiferromagnetic (AF) structures ${ }^{6,7}$ and incommensurate spin-density waves. ${ }^{8,9}$

Very recently, soft-x-ray resonant magnetic reflectivity $(\mathrm{XRMR})^{10}$ experiments combined with new first-principles calculations ${ }^{11}$ on Fe films have indicated that the spin structure for 6 and 8 ML thick films can be written as $[\uparrow \uparrow \downarrow][\uparrow \downarrow][\uparrow]$ and $[\uparrow \uparrow \downarrow][\uparrow \downarrow][\uparrow \downarrow][\uparrow]$, from the top layer to the bottom (at the $\mathrm{Fe} / \mathrm{Cu}$ interface). The calculations were based on a slab geometry where the lattice parameter of an isotropic fcc-Fe film was chosen equal to the lattice parameter of bulk $\mathrm{Cu} .{ }^{11} \mathrm{In}$ the XRMR experiment, the films were grown by pulsed laser deposition (PLD), ${ }^{10}$ which leads to improved layer-by-layer growth, producing an isotropic fcc structure. ${ }^{12,13}$ The same does not hold for films grown by thermal deposition (TD), which exhibits several complex reconstructions ${ }^{13}$ that can affect the magnetic properties.

The calculations suggest that the magnetic structure consists of robust blocks of collinear spins, whereas the relative orientation of the spins of different blocks can easily vary. The upper block contains two FM coupled layers followed by one AF coupled layer. Further blocks contain pairs of AF coupled layers. In the schematic representations of the spin structure of 6- and 8-ML-thick films (see above), the blocks are separated by brackets. The grouping into the spin blocks became evident by the study of the inverse spin reorientation (SRT) from in-plane to out-of-plane, ${ }^{10}$ which takes place between about 6 and 8 ML. ${ }^{12}$ The XRMR study provided evidence that within the film, the SRT proceeds from the top of the film to the bottom, i.e., the upper spins are oriented out-of-plane before those at the bottom of the film. The XRMR results were in good agreement with the model proposing that the blocks rotate as rigid units relative to each other during the SRT. ${ }^{10}$ The physical reason for the formation of the block structure was found in the hierarchy of exchange interactions, which are much stronger within the blocks than between the blocks.

Recently, Sandratskii ${ }^{11}$ has shown that this complex hierarchy of exchange interactions can lead to strongly nonuniform temperature dependence of the magnetization across the film: the temperature dependence of magnetization $m_{\mu}(T)$ can be very different for different layers $(\mu)$. The purpose of this paper is to explore this question experimentally.

Direct experimental access to layer-resolved magnetization is very difficult. The classical analytical methods such as magneto-optical Kerr effect (MOKE) and x-ray magnetic circular dichroism (XMCD) experiments provide information only on the magnetic moments averaged over the film. Some attempts to establish the temperature dependence of the magnetization profile by MOKE measurements were made ${ }^{4,9}$ by comparison between data collected for TD grown Fe films with different thicknesses. The authors came to the conclusion that the ordering temperature of the surface layers is about $300 \mathrm{~K}$ and is therefore higher than the $\mathrm{AF}$ ordering temperature of the internal layers, which is about 200-220 K. This value, however, was challenged by Mössbauer spectroscopy measurements, ${ }^{14}$ which show a fingerprint of the AF state only at temperatures below $70 \mathrm{~K}$. In the MOKE experiments, the strategy used in the estimation of the AF ordering temperature assumes that internal layers of the films with different thicknesses respond in the very same way to the variation of temperature. For ultrathin films whose magnetic properties are known to be very sensitive to the number of layers, such an assumption is questionable. Therefore, the 
analysis of the temperature dependence of the magnetic profile of a single film is highly desirable.

In this context, soft XRMR has proven its capability to study depth-resolved spin structures, provided that reflection intensities can be collected up to high scattering angles. ${ }^{10,15-18}$ To this end, we have carried out a soft-XRMR study on a 6-MLthick Fe film grown on $\mathrm{Cu}(001)$ using pulsed laser deposition (PLD). Our study provides evidence for an inhomogeneous temperature dependence of magnetization within the film in the temperature regime between 60 and $300 \mathrm{~K}$.

The remainder of the paper is organized as follows. The details of the XRMR measurements are described in Sec. II. In Sec. III, we present the experimental results and their analysis. A summary is given in Sec. IV.

\section{EXPERIMENT}

Prior to the film growth, the $\mathrm{Cu}(001)$ surface was cleaned under UHV conditions by repeated cycles of $1 \mathrm{keV}$ Ar-ion sputtering followed by annealing at $870 \mathrm{~K}$. The surface composition was checked by Auger electron spectroscopy (AES) and the long-range order was probed by low-energy electron diffraction $($ LEED), showing sharp $(1 \times 1)$ spots. Scanning tunneling microscopy (STM) images indicated terrace sizes as large as $200 \mathrm{~nm}$. The 6-ML-thick Fe film was then deposited on $\mathrm{Cu}(100)$ at room temperature by PLD using a $\mathrm{KrF}(248 \mathrm{~nm})$ laser as reported in Ref. 12. At this coverage, longitudinal MOKE measurements show a hysteresis loop even at $T=$ $290 \mathrm{~K}$ (Tc was found to be very close to room temperature, $\mathrm{Tc} \approx 308 \mathrm{~K})$. After $\mathrm{Fe}$ deposition, the sample was capped by a 4.5-nm-thick Au film, which did not affect the magnetic properties, as deduced from the MOKE experiments.

Soft-XRMR experiments were carried out at the Surface/Interfaces: Microscopy (SIM) beam line ${ }^{19}$ of the Swiss Light Source, Paul Scherrer Institute, Villigen, Switzerland, using the UHV diffractometer outlined in Ref. 20. The reflectivity signal $(I)$ was collected in the $\theta-2 \theta$ scan mode using the photon energy tuned to the $L_{2}$ and $L_{3}$ absorption edges of Fe. Figure 1(a) schematically shows the experimental geometry. The sample was magnetized in situ by a permanent magnet $\left(\mu_{0} H=0.2 T\right)$ followed by data collection in remanence. This procedure was repeated for each investigated temperature $(T)$. Taking into account a low-saturation field and high remanence of the in-plane magnetization, ${ }^{12}$ we have chosen to probe the magnetization by using the longitudinal scattering geometry. The external magnetic field was applied along the [110] direction of the $\mathrm{Cu}(001)$ crystal. The measurements were carried out in the temperature range between 60 and $300 \mathrm{~K}$.

Both circularly and linearly polarized light was used to probe the layer-dependent orientation of the magnetic moments. XRMR is a photon-in/photon-out technique, and both polarization states can be used to probe different directions of the magnetic moments, as discussed by Hill and McMorrow, ${ }^{21}$ and evidenced in the soft-x-ray range through the first reflectivity measurements. ${ }^{22-24}$ In the present case, considering only the dipolar $2 p \rightarrow 3 d$ transition, the atomic scattering factor can be written as $f=-\left(\mathbf{e}_{f} \cdot \mathbf{e}_{i}\right) F_{c}-\left(\mathbf{e}_{f} \cdot \mathbf{e}_{i}\right) \cdot \hat{\mathbf{m}} F_{m}$, where the second-order term in $\hat{\mathbf{m}}$ is neglected. The parameter $\mathbf{e}_{i}\left(\mathbf{e}_{f}\right)$ is the polarization vector of the incoming (scattered) beam, and $\hat{\mathbf{m}}$ denotes the (local) magnetization direction of the $\mathrm{Fe}$ (a)

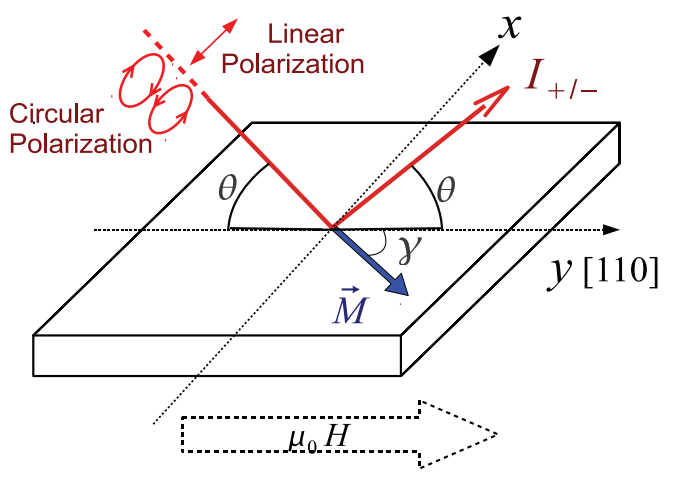

(b)

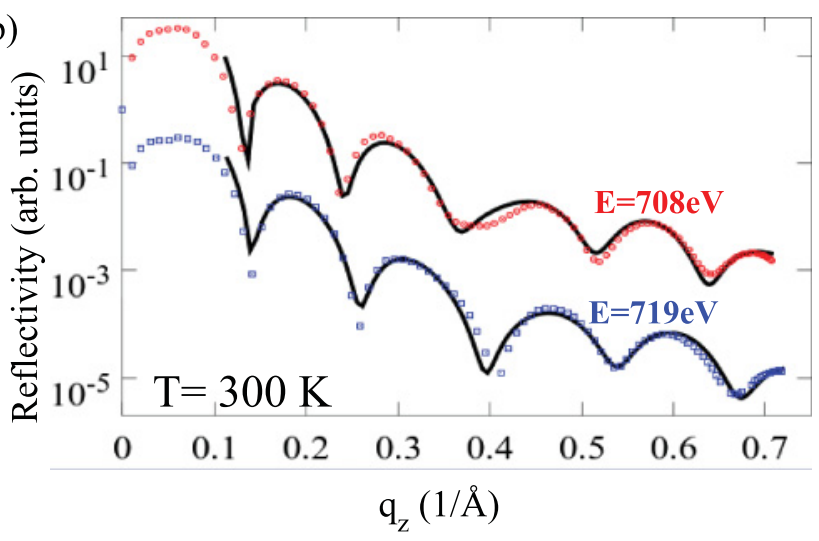

FIG. 1. (Color online) (a) Schematic of the experimental geometry. The sample is magnetized along the [110] direction, parallel to the $y$ axis of the coordinate frame (longitudinal geometry). The angle $\theta$ characterizes the incidence angle of the light relative to the sample plane. The angle $\gamma$ corresponds to the deviation of the magnetization vector $M$ from the $y$ axis. (b) Experimental (symbols) and calculated (solid line) specular reflectivity at two different photon energies at the $\mathrm{Fe} L_{3}$ and $L_{2}$ absorption edge. The maximum scattering angle $(2 \theta)$ is equal to $160^{\circ}$.

atoms. $F_{c}$ and $F_{m}$ are complex terms representing charge and magnetic scattering, respectively. $F_{c}$ includes the Thomson scattering and resonant corrections terms. The imaginary part of $F_{m}$ is related to the magnetization dependence of the absorption, which is derived from XMCD experiments. Note that by using linearly $\pi$-polarized incident light, an asymmetry in the second term of the scattering factor, and consequently in the reflectivity, is observed only upon reversal of the transverse magnetization. This is because no Kerr rotation is expected under this condition and the $\left(\mathbf{e}_{f} \cdot \mathbf{e}_{i}\right)$ orientation is constant, resulting in a nonzero asymmetry due to the sign reversal of $\hat{\mathbf{m}}$. From here it follows that the reflectivity in the $\pi-\pi$ scattering channel solely depends on the transverse magnetic component $\left(m_{x}\right)$, while the magnitude of the $\pi-\sigma$ channel is constant upon magnetization reversal, ${ }^{21}$ which allows the use of linearly $\pi$-polarized incident light for an independent analysis of $m_{x}$.

Intensities for right and left circular polarized light $[I(+)$ and $I(-)$ ] were collected by inversion of the beam polarization to derive the asymmetry defined by $A\left(q_{z}\right)=[I(+)-$ $I(-)] /[I(+)+I(-)]$, where $q_{z}$ is the momentum transfer normal to the sample surface given by $q_{z}=2 k \sin (\theta)$, and $k$ is the amplitude of the photon wave vector. Experiments 
with $\pi$-polarized incident light were carried out by reversing the magnetic field. For circularly polarized light $(c)$, the asymmetry can be expressed as $A^{c}=A^{c}\left(q_{z}, m_{y}, m_{z}\right)$ with a small dependence on $m_{x}$ at the denominator of the ratio, whereas for the linearly $\pi$-polarized light, the asymmetry takes the form $A^{\pi}=A^{\pi}\left(q_{z}, m_{x}\right)$. In the absence of longitudinal and polar components $\left(m_{y}=m_{z}=0\right), A^{c}=0$; in the absence of a transverse component $\left(m_{x}=0\right), A^{\pi}=0$. In Fig. 1(a), the vector $M$ represents the effective magnetization over the area illuminated by the $\mathrm{x}$-ray beam $\left(2 \times 2 \mathrm{~mm}^{2}\right)$. The angle $\gamma$ is used as a fitting parameter quantifying the total projection of the magnetization along the the longitudinal $(y)$ and transverse $(x)$ directions.

\section{RESULTS AND DISCUSSION}

\section{A. Geometric film structure}

The geometric film structure is determined on the basis of the average reflectivity given by $\bar{I}=[I(+)+I(-)] / 2$. The symbols in Fig. 1(b) represent $\bar{I}$ versus $q_{z}$ collected at two different photon energies close to the Fe $L_{3}$ and $L_{2}$ absorption edges. Solid lines show the best fit to the measured data based on the structure model given in the left panel in Fig. 2. An important difference to the idealized model structure used in Ref. 11 is a mixed $\mathrm{Fe}_{x} \mathrm{Cu}_{(1-x)}$ alloy layer, which is described by introducing a $\mathrm{Fe}_{x} \mathrm{Cu}_{(1-x)}$ slab with a homogeneous concentration $(x)$ at the $\mathrm{Fe} / \mathrm{Cu}$ interface. It simulates the effects of intermixing and roughness on the reflectivity, since these effects are indistinguishable by this technique due to the lack of lateral resolution.

It is assumed that the complex refractive index $(N)$ of the intermixed region depends linearly on the refractive indices of constituting elements $N_{1}=x N_{\mathrm{Fe}}+(1-x) N_{\mathrm{Cu}}$, where $x$ is the concentration of $\mathrm{Fe}$. The concentration $x$ is refined simultaneously with other structural parameters given in Table I.

The nominally 6-ML-thick Fe film between the Au-capping layer and the $\mathrm{Cu}$ bulk was then modeled by a Fe slab, representing five Fe layers, and one intermixed layer, representing the $\mathrm{Fe} / \mathrm{Cu}$ interface layer. The thickness of the $\mathrm{Fe}$ slab is

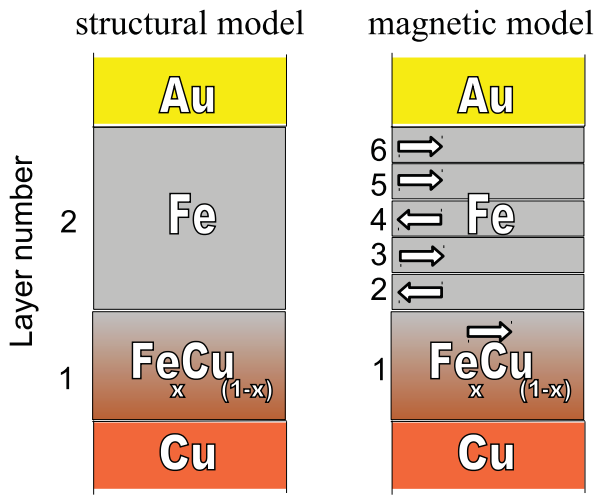

FIG. 2. (Color online) Schematic structure model (left) of the nominally 6-ML-thick Fe film on $\mathrm{Cu}(001)$. The intermixing between the $\mathrm{Fe}$ and $\mathrm{Cu}$ substrate is taken into account by the $\mathrm{Fe}_{x} \mathrm{Cu}_{(1-x)}$ layer (No. 1). The basic magnetic structure is shown on the right. Arrows indicate the direction of the magnetic moments in each layer. allowed to vary from $5 \times[1.78$ to $1.82 \AA] .{ }^{25}$ The thickness of the intermixed layer was found to be $5.80 \pm 0.20 \AA$ and the Fe concentration was equal to $x=50 \pm 5 \%$. The estimated thickness of the intermixed layer is compatible with XRMR measurements at $\mathrm{Cu} L_{2,3}$ edges of another sample preparation (not shown), which indicates an induced polarization in $\mathrm{Cu}$ atoms homogeneously distributed over 5.0 $\pm 0.4 \AA$. More complicated structure models, e.g., involving some concentration gradient within the intermixed layer, were not considered. This is because of the limited electron-density contrast between $\mathrm{Fe}$ and $\mathrm{Cu}$ leading to ambiguous results going in parallel with no significant improvement of the fit quality. The right panel of Fig. 2 shows the magnetic structure of the film as derived from the XRMR measurements, which will be discussed below.

\section{B. Magnetic structure and its temperature dependence}

The determination of the layer-resolved magnetic structure of the film and its temperature dependence is based on the fit of the calculated asymmetry $\left[A_{\text {calc }}\left(q_{z}\right)\right]$ to the experimental one, $A_{\text {exp }}\left(q_{z}\right)$. The evaluation of $A_{\text {calc }}\left(q_{z}\right)$ was carried out by using the $4 \times 4$ matrix algorithm developed for MOKE calculations in multilayer systems. ${ }^{26,27}$ The energy-dependent complex refractive index, $N_{\mathrm{Fe}}(E)$ and the optical constant, $Q_{\mathrm{Fe}}(E)$ of $\mathrm{Fe}$ were derived from $\mathrm{x}$-ray absorption and XMCD measurements on a bulk $\mathrm{Fe}$ film, ${ }^{28}$ which allow the determination of the imaginary components of the charge and magnetic scattering length. The corresponding real parts are calculated by using the Kramers-Kronig transformation. Values of $Q_{\mathrm{Fe}}(E)$ correspond to magnetically saturated Fe for which the sum rules yield an atomic magnetic moment of $m_{\circ}=2.1 \mu_{B} .{ }^{28}$ In our model, the layer magnetic moments are expressed in units of $m_{\circ}$, and $m=1$ corresponds to the magnetic moment of $2.1 \mu_{B}$ per $\mathrm{Fe}$ atom.

The magneto-optic constant is assumed to scale linearly with the value of the magnetization $Q(E)=|m| \cdot Q_{\mathrm{Fe}}(E)$. Thus, for the intermixed layer (No. 1), the magneto-optic constant takes the form $Q_{1}(E)=\left|m_{1}\right| \cdot x \cdot Q_{\mathrm{Fe}}$ since it is proportional to the Fe concentration.

The first result of this study is that the magnetic structure can be characterized by two FM coupled layers at the top, followed by one AF coupled layer. Deeper layers form blocks of AF coupled layers. This model reproduces the results of the previous analysis. ${ }^{10}$ As has been already shown there, the fit quality is very sensitive to the specific model of the magnetic structure. For instance, the symbols in Fig. 3 represent the experimentally derived asymmetry $\left[A^{c}\left(q_{z}\right)\right]$ collected at $T=$ $200 \mathrm{~K}$. The lines represent two fits using the spin structure models which are shown in the upper part of the figure.

In the first one (left, red dashed line), a homogeneous (FM) spin structure throughout the film is assumed, whereas the second one (right, blue solid line) corresponds to the spin structure $[\uparrow \uparrow \downarrow][\uparrow \downarrow][\uparrow]$ as found previously, ${ }^{10}$ and additionally involving the (magnetic) mixed interface layer. Numbers indicate the magnitude of the magnetic moments $m$ in units of $m_{\circ}$, while the arrows indicate the direction of the magnetic moment within each layer. For the FM model on the left, the best fit that can be achieved is shown, but very large deviations between the calculated and experimental $A^{c}\left(q_{z}\right)$ are 
TABLE I. Structure parameters for $6 \mathrm{ML} \mathrm{Fe} / \mathrm{Cu}(001)$. The $\mathrm{Cu}$ substrate density was kept fixed to its bulk value and the Fe layer thickness was allowed to vary from 5 x [1.78 to $1.82 \AA]$. In the fourth column the refined values for the roughness is listed computed as the standard deviation from the interface composition and the alloy concentration $(x)$ in the $\mathrm{Fe}_{x} \mathrm{Cu}_{(1-x)}$ alloy layer are listed. The roughness is computed as the standard deviation from the interface position.

\begin{tabular}{lcrc}
\hline \hline Medium & Thickness $(\AA)$ & Density $\left(\mathrm{g} / \mathrm{cm}^{2}\right)$ & Roughness $(\AA)$ \\
\hline $\mathrm{Au}$ & $45.5 \pm 0.5$ & $19.9 \pm 0.6$ & $\sigma_{\mathrm{Air} / \mathrm{Au}} 1.7 \pm 0.3$ \\
$\mathrm{Fe}$ & $5 \times[1.78$ to 1.82$]$ & $7.8 \pm 0.7$ & $\sigma_{\mathrm{Au} / \mathrm{Fe}} 0.3 \pm 0.3$ \\
$\mathrm{Fe}_{x} \mathrm{Cu}_{1-x}$ & $5.80 \pm 0.20$ & & $x=0.50 \pm 0.05$ \\
$\mathrm{Cu}$ & $\infty$ & 8.96 & \\
\hline \hline
\end{tabular}

still observed. By contrast, the spin structure $[\uparrow \uparrow \downarrow][\uparrow \downarrow][\uparrow]$ reproduces the experimental data very well. Deviations are observed only at places associated with a steep gradient in the asymmetry, which, e.g., might be attributed to errors in the photon energy, finite experimental resolution, or uncertainties in the detailed geometric film structure.

In general, we find that good fits to the experimental asymmetry are only possible by assuming the spin structure as shown in the right panel of Figs. 2 and 3. The slices are numbered from $\mu=1$ for the $\mathrm{Fe}_{x} \mathrm{Cu}_{(1-x)}$ alloy layer to $\mu=6$ for the Fe layer next to the Au-capping layer. In this way, we follow the labeling used in Ref. 11.

In principle, the direct fit of the asymmetry curves, as shown in Fig. 3, using a layer-resolved (temperature-dependent) magnetization model is possible; however, this procedure suffers from a large number of fit parameters and correlations between them, which in turn leads to large uncertainties. For this reason, we followed a different path, which significantly reduces both the number of fit parameters and the correlation problem by modeling the system in terms of a Heisenberg Hamiltonian of interacting moments similar to the Hamiltonian used in Ref. 11. The fit parameters in this approach are

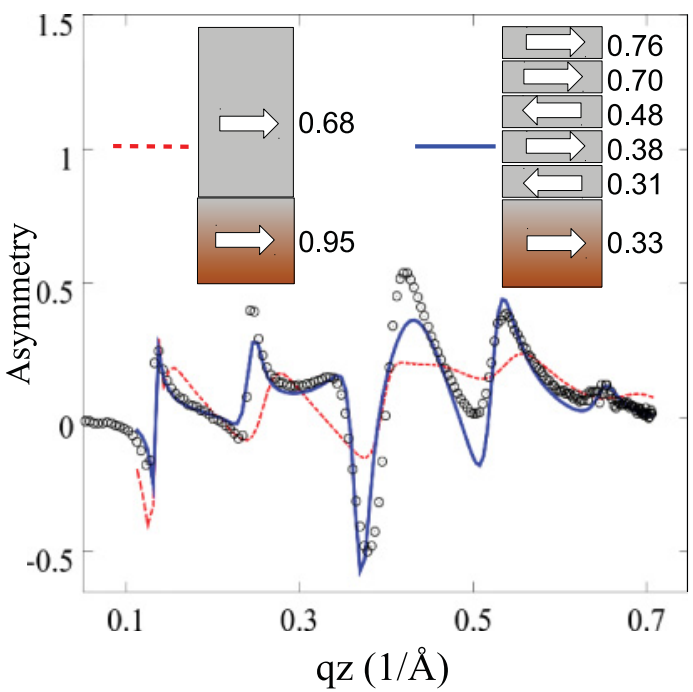

FIG. 3. (Color online) Experimental (symbols) and calculated (lines) asymmetry, $A^{c}\left(q_{z}\right)$, for circular polarization of the incident light collected at the $\mathrm{Fe} L_{3}$ absorption edge (708 eV) at $T=200 \mathrm{~K}$. A schematic corresponding to two different magnetic structure models is shown. Numbers indicate the magnitude of the magnetic moments $m$ in units of $m_{\circ}$. The red (dashed) and blue (solid) lines are related to the magnetic structure models as indicated. the temperature-independent Heisenberg exchange parameters $J_{\mu \nu}$. Within the mean-field approximation (MFA), the layerresolved temperature dependence of the magnetization is given by the following system of self-consistent equations:

$$
\left\langle m_{\mu}(T)\right\rangle=L\left[1 /(k T) \sum_{\nu} J_{\mu \nu}\left\langle m_{\nu}\right\rangle\right],
$$

where $\left\langle m_{\mu}\right\rangle$ is the magnetization of layer $\mu$.

$J_{\mu \nu}$ corresponds to the exchange parameter between layers $\mu$ and $\nu$. The intralayer exchange parameters $(\mu=\nu)$ give the total exchange field produced by the atoms of the given layer at the position of a selected atom of this layer. ${ }^{11} L(a)$ represents the Langevin function: $L(a)=\operatorname{coth}(a)-1 / a$. For the interlayer exchange parameters $(\mu \neq v)$, only nearestlayer interactions are taken into account. The intralayer exchange parameter of the surface and interface layer are represented by $J_{11}$ and $J_{66}$, respectively; the intralayer exchange parameter of the inner layers is represented by the single fit parameter $J_{22-55}$. The set of exchange parameters was refined to simultaneously fit the complete data set $A\left(q_{z}, T\right)$ to the simulated asymmetries by using the layer magnetization $\left\langle m_{\mu}(T)\right\rangle$ obtained from Eq. (1).

The symbols in Fig. 4 represent the experimental asymmetries $A\left(q_{z}\right)$ collected at different photon energies at the Fe $L_{3}$ edge for circular $(c)$ and linear $(\pi)$ polarization of the incident light. Data were collected between 60 and 300 K. Direct inspection of the curves in Fig. 4 indicates that the samples are magnetized in-plane, since the asymmetry curves $A^{c}\left(q_{z}\right)$ tend to zero at high angles $\theta$, which can be directly related to the absence of the out-of-plane component of magnetization. ${ }^{29}$ The nonzero asymmetry in the case of linearly $\pi$-polarized incident light, $A^{\pi}\left(q_{z}\right)$ [red lines, labeled by $(\pi)$ ], reveals the presence of a nonzero transverse component of the magnetization $m_{x}$. The increase of the $A^{\pi}\left(q_{z}\right)$ amplitude as the temperature decreases is a consequence of the increasing of the transverse component $m_{x}$, which is explained by the presence of a preferential magnetization axis in the film plane that is not aligned to the field direction $(y)$; this will be discussed in the next section.

In this context, considering the absence of the out-of-plane component and the presence of the in-plane easy axes, the magnetization directions of different layers in Eq. (1) are constrained to be in-plane and collinear to each other. ${ }^{30}$ It should be noted that the effect of magnetic anisotropy is not taken into account by Eq. (1), but it was observed in the experiment by the (temperature-dependent) variation of the transverse component $m_{x}$ (see the next section for details). 


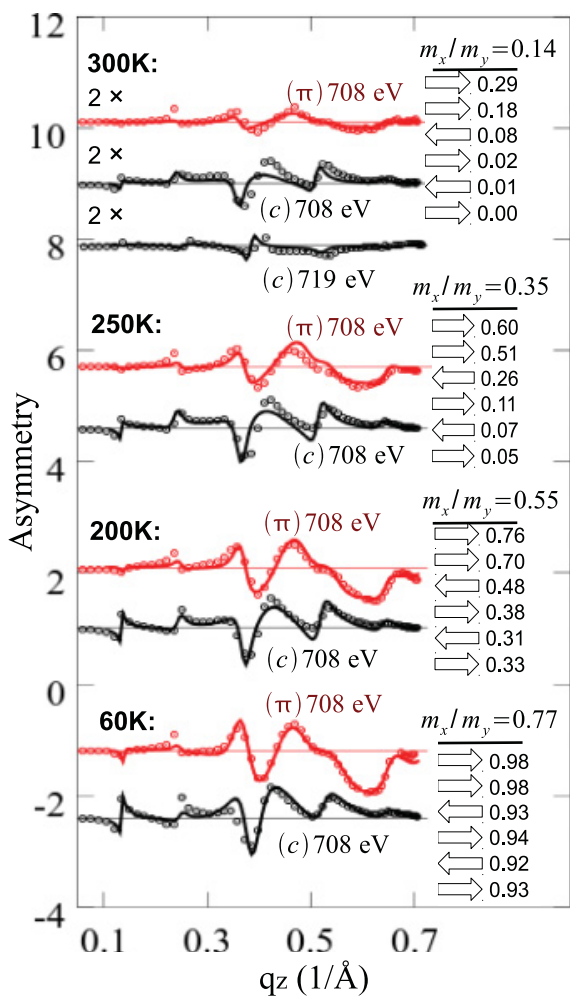

FIG. 4. (Color online) Temperature-dependent experimental (symbols) and calculated (lines) asymmetry, $A\left(q_{z}\right)$, for circular $(\sigma)$ and linear $(\pi)$ polarization of the incident light collected at different energies in the vicinity of the Fe $L_{3}$ absorption edge. The lines are vertically shifted from the zero value for clarity. Asymmetries for the $300 \mathrm{~K}$ data are shown enlarged by a factor of two. A schematic of the corresponding magnetic structure models is shown on the right. Numbers indicate the magnitude of the magnetic moments $m$ in units of $m_{\circ}$. The ratio $\left(m_{x} / m_{y}\right)$ is indicated for each temperature.

The solid lines in Fig. 4 represent $A_{\text {calc }}\left(q_{z}\right)$ based on the magnetic structures schematically shown on the right for temperatures between 60 and $300 \mathrm{~K}$. Very good agreement between $A_{\text {calc }}\left(q_{z}\right)$ and $A_{\text {exp }}\left(q_{z}\right)$ is obtained in the whole investigated temperature range. As in Fig. 3 , the arrows indicate the direction of the magnetization within each layer. The numbers at the arrows give the values of the modulus of magnetic moments $|m|$ of the layers in units of $m_{\circ}$. They are derived from Eq. (1) by using the refined exchange parameters. For each temperature, the ratio $m_{x} / m_{y}$ is also indicated, where $m_{x}^{2}+m_{y}^{2}=|m|^{2}$. It is derived from the relationship between the amplitudes of $A^{c}\left(q_{z}\right)$ and $A^{\pi}\left(q_{z}\right)$.

In the following, the most important results are summarized:

(i) We find a significant layer dependence of both the intralayer exchange parameters $\left(J_{\mu \mu}\right)$ and the interlayer exchange parameters $\left(J_{\mu, \mu+1}\right)$. For the intralayer exchange parameters, we obtain $J_{11}=33 \pm 6, J_{22-55}=11 \pm 3$, and $J_{66}=45 \pm 3 \mathrm{meV}$. This result is in qualitative agreement with available theoretical predictions: Spišák et al. ${ }^{7}$ and Sandratskii ${ }^{11}$ report much stronger intralayer exchange interactions for the surface and interface layers than for the inner layers. Figure 5 shows the interlayer exchange parameters $\left(J_{\mu, \mu+1}, \mu=1, \ldots, 5\right)$. The large positive value of $J_{56}$ reflects

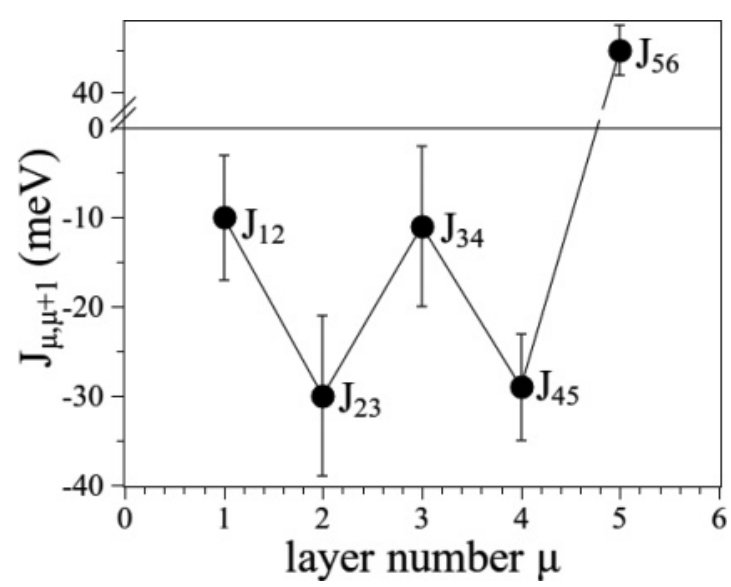

FIG. 5. Experimentally derived temperature-independent interlayer exchange parameters $\left(J_{\mu, \mu+1}\right)$ in $6 \mathrm{ML} \mathrm{Fe} / \mathrm{Cu}(001)$.

the strong FM coupling between the two topmost layers, while the negative ones $\left[J_{\mu, \mu+1}, \mu=1-4\right]$ correspond to the AF coupling between the deeper layers. Furthermore, the latter can be separated into two groups, namely, one group characterized by a large magnitude of the exchange parameters $\left(J_{23}\right.$ and $\left.J_{45}\right)$ and a second group with smaller magnitudes $\left(J_{12}\right.$ and $J_{34}$ ). This reflects the hierarchy of exchange interactions suggested in Refs. 11 and 10, which is the physical basis for the formation of the spin blocks in the magnetic structure of fcc- $\mathrm{Fe} / \mathrm{Cu}(001)$ films.

(ii) In agreement with recent experimental and theoretical studies (Refs. 10 and 11), we find that the magnetic structure of the film is characterized by the two topmost FM coupled layers followed by an AF coupled third layer. The deeper two layers are strongly AF coupled forming the film structure $\uparrow \uparrow \downarrow|\uparrow \downarrow| \uparrow$ (from top to bottom).

(iii) The uncertainties for the $J_{\mu \mu}$ and $J_{\mu, \mu+1}$ lie in the $\pm 5-10 \mathrm{meV}$ range. We have carried out extensive calculations to obtain a precise insight into the dependence of the fit quality on the correlations between different parameters. As an example, Fig. 6 shows two contour plots in which the relative variation of the residuum, $\Delta R_{u}=\left(R_{u}-R_{u}^{\min }\right) / R_{u}^{\min }$ is plotted versus $J_{12}$ and $J_{23}$ as well as versus $J_{34}$ and $J_{45}$. Both pairs contain one strong and one weak exchange interaction parameter. The residuum $R_{u}$ is defined as $R_{u}=\sum \mid A_{\text {calc }}-$ $A_{\text {exp }}\left|/ \sum\right| A_{\text {exp }} \mid$, where the summation extends over all data points. The calculations indicate a strong dependence of $R_{u}$ on $J_{\mu \nu}$ (Fig. 6). The contour lines represent isolines of constant $\Delta R_{u}=5,10,20 \ldots \%$ (see labels). We have chosen the 5\% level as an estimate for the uncertainty of $J_{\mu \nu}$.

(iv) Using the exchange parameters for which the best fit is obtained, it is possible to calculate the temperature dependence of the magnetization for each layer according to Eq. (1). Figure 7 shows $m_{\mu}(T)$ for $\mu=1, \ldots, 6$. Negative values indicate the antiferromagnetic orientation of $m$. We find an overall transition temperature, $T_{c}=308 \mathrm{~K}$, but the temperature dependence of the magnetization of each layer certainly depends on its position within the film. This is the most important result of this study. It represents a remarkable result in that it probes the inhomogeneity of the temperature- 


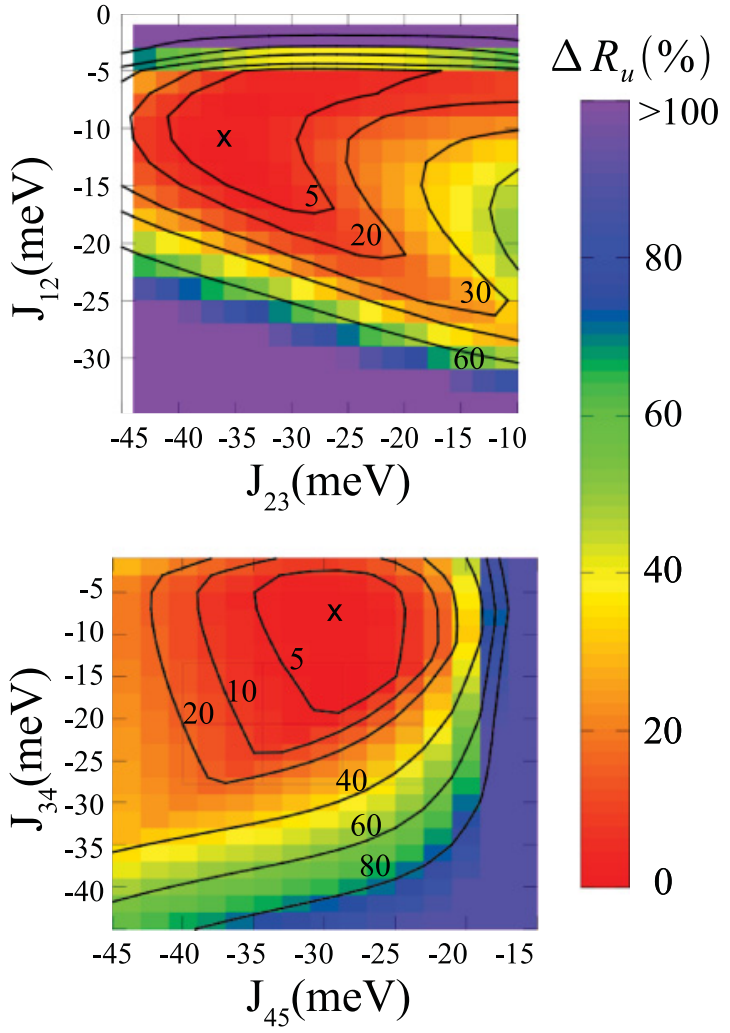

FIG. 6. (Color online) Contour plots of $\Delta R_{u}$ vs (a) $J_{12}, J_{23}$ and (b) $J_{34}, J_{45}$. The color code is shown on the right. Crosses mark the minima. The lines represent isolines of constant $\Delta R_{u}=$ $5,10,20 \ldots \%$ as labeled.

dependent magnetization in a film with few atomic layers using a layer-resolved analytical tool.

In detail, there is a large difference between the $m_{\mu}(T)$. The "bulklike" (convex) temperature profile of $m_{5}$ and $m_{6}$ is related to the strong ferromagnetic coupling between the upper layers $\left(J_{56}\right)$ and the strong intralayer exchange interaction in the topmost layer. By contrast, the temperature dependence of the magnetization of deeper layers $(\mu=1,2,3,4)$ above about $220 \mathrm{~K}$ has an induced character. ${ }^{31}$ This is the consequence of the weaker exchange interaction between the atoms of these layers. The total magnetization $[M(T)]$ is given by the sum of the layer contributions and is dominated by the magnetization of the topmost layers. $M(T)$ is shown in the inset of Fig. 7. Upon cooling from $T_{c},\left|m_{\mu}\right|(\mu=1-4)$ initially increases slowly, followed by a region characterized by a steep increase below about $220 \mathrm{~K}$. In consequence, this leads to a steplike feature in the total magnetization at $220 \mathrm{~K}$ (see arrows in Fig. 7), which has been observed in the MOKE experiments of Qian et al. ${ }^{9}$

The inhomogeneity of the temperature dependence of the magnetization within the film as a consequence of the hierarchy of exchange interactions is in correspondence with the theoretical considerations reported previously (see Figs. 7 and 8 in Ref. 11). The only difference between experiment an theory is related to $m_{1}(T)$ (convex versus concave profile), which is tentatively attributed to the $\mathrm{Fe} / \mathrm{Cu}$ intermixing. Note that the $\mathrm{Fe} / \mathrm{Cu}$ intermixing simulated

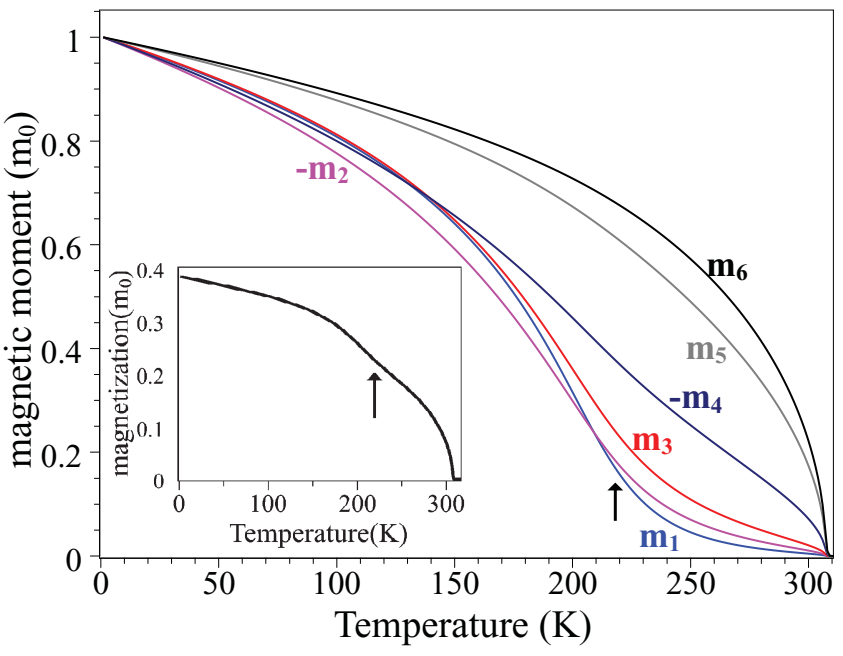

FIG. 7. (Color online) Layer resolved magnetic moments (in units of $\mathrm{m}_{0}$ ) vs temperature derived from Eq. (1) using the exchange parameters shown in Fig. 4. The inset presents the temperature dependence of the total magnetization $(M)$. Arrows mark the steplike increase of $M$ at about $220 \mathrm{~K}$.

for an $8 \mathrm{ML}$ film leads to the behavior of the interface magnetization similar to the behavior obtained in our study. ${ }^{11}$

(a)

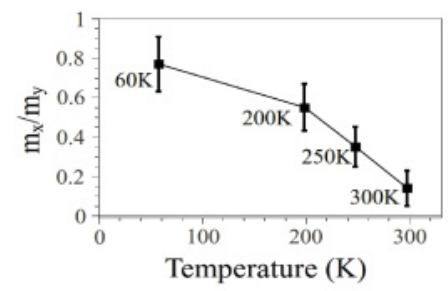

(b)

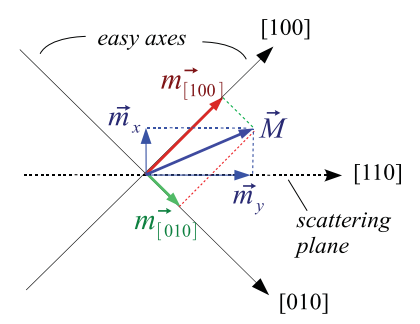

(c)

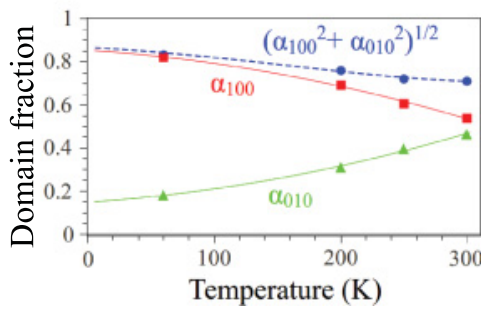

FIG. 8. (Color online) (a) Ratio between the projections of the magnetization along the transverse $\left(m_{x}\right)$ and longitudinal $\left(m_{y}\right)$ directions. (b) The lengths of the arrows along [010] and [100] present the contributions of the domains with corresponding easy axes. (c) Temperature dependence of the domain distribution $\alpha_{100}$ (red line) and $\alpha_{010}$ (green line), and the $\zeta$ factor (dashed blue line). 


\section{Temperature dependence of the transverse component of the magnetization}

The collection of reflectivity data using linearly $\pi$-polarized incident light allows the direct analysis of the transverse component $m_{x}$ of the magnetization. The sample was magnetized along the [110] direction ( $y$ axis; see Fig. 1), while the collection of the asymmetry curves, $A^{\pi}\left(q_{z}\right)$, was carried out at remanence. Nonzero asymmetries were measured by reversing the field, leading us to the conclusion that the transverse component $\left(m_{x}\right)$ is also flipped when the applied longitudinal magnetic field is reversed. As can be seen directly from the measured asymmetry curves in Fig. 4, there is always a finite component $m_{x}$, which increases with decreasing temperature.

The behavior of the transverse component $m_{x}$ is interpreted by the presence of two domains, in which the magnetization is oriented parallel to two different but symmetry-equivalent easy magnetization directions, which are [100] and [010]. The flipping of $m_{x}$ is then a consequence of the magnetic reversal of these domains. The ratio between transverse and longitudinal components $\left(m_{x} / m_{y}\right)$ is actually a measure of the relative fraction of the two domains. Figure 8(a) shows the dependence of the ratio $\left(m_{x} / m_{y}\right)$ on temperature. Figure $8(\mathrm{~b})$ outlines the model, in which the magnetization $M$ represents the sum over the two magnetizations $m_{[100]}$ and $m_{[010]}$, while $m_{x}$ and $m_{y}$ are the accessible quantities in the experiment. The fraction of the domains is represented by $\alpha_{100}$ and $\alpha_{010}$, such that $\alpha_{100}+\alpha_{010}=1$. In an ideal case in which the magnetic field is applied exactly parallel to the [110] axis, there should be an equal fraction of [100] and [010] oriented domains: $\alpha_{100}=\alpha_{010}$. Consequently, in this case, the total magnetization is parallel to [110]. We find experimentally that the domains do not have an equal weight, i.e., $\alpha_{100} \neq \alpha_{010}$, and their ratio is temperature dependent. From the ratio $\left(m_{x} / m_{y}\right)$, we derived the temperature dependence of $\alpha_{100}$ and $\alpha_{010}$, which is shown in Fig. 8(c).

The temperature-dependent domain redistribution is a subtle effect which depends on a possible misalignment of the applied field with respect to the [110] direction, and, more importantly, on the distribution of defects in the film that can act as pinning centers. It can be expected that the influence of such defects is stronger at low temperature. This agrees with the estimated temperature dependence of the domain distribution that tends to equal occupation for $T \longrightarrow T_{c}$ [see Fig. 8(c)]. The study of such processes, however, is outside of the scope of the paper.

Finally, two issues should be emphasized. First, it is important that the experimental technique of XRMR allows one to disentangle different components of the magnetization and, in principle, opens the avenue for the study of the effects of magnetic anisotropy and domain redistribution. Second, the derivation of the temperature dependence of the magnetization outlined in the last section continues to hold also under the presence of two domains. Providing that, in comparison to the exchange interaction, the influence of the dipole-dipole interaction between domains on the temperature dependence is negligible, Eq. (1) remains valid within each domain. In this case, the total layer magnetization would be reduced by a factor $\zeta(T)=\left(\alpha_{100}^{2}+\alpha_{010}^{2}\right)^{1 / 2}$, which is shown in Fig. 8(c). The temperature dependence of $\zeta(T)$ is much weaker than the temperature dependence of the magnetization and can be safely neglected. The fit paramerers $\left(J_{i i}\right.$ and $\left.J_{i j}\right)$ calculated for a single-domain model can thus be directly transferred to the multiple-domain model in general, and to the two domain model in particular by a simple rescaling of the values of magnetic moments in order to compensate the reduction factor $\zeta$. The magnetic moment of $\mathrm{Fe}$ atoms in the two-domain model should be somewhat higher than the values assigned in the single-domain model. The main physical conclusions discussed in Sec. III B remain valid.

\section{SUMMARY}

In summary, we have presented a soft-XRMR study of the layer-resolved temperature dependence of the magnetic structure in a $6 \mathrm{ML}$ fcc-Fe film grown on $\mathrm{Cu}(001)$. The analysis is based on the Heisenberg Hamiltonian using a small number of temperature-independent exchange parameters. The experimentally derived exchange parameters confirmed the physical picture suggested in our previous work involving a hierarchy of the exchange interactions leading to the formation of blocks of layers with robust magnetic structure whereas the exchange interaction between different blocks is relatively weak. Clear evidence is found for an inhomogeneous temperature-dependent magnetization profile within the film. While the two topmost FM coupled layers exhibit a convex quasibulklike $m(T)$ profile with a rapid onset of $m$ below $T_{c}$, deeper layers exhibit induced magnetization character with $m(T)$ slowly increasing with decreasing temperature. A steep increase of the magnetization in deeper layers happens at temperatures between 200 and $250 \mathrm{~K}$; this range coincides with the $\mathrm{AF}$ ordering temperature estimated from MOKE experiments in TD films. ${ }^{4,9}$

The collection of reflectivity data using linearly $\pi$-polarized incident light indicates the presence of a transverse remanent magnetization component. The temperature dependence of the transverse component is interpreted as resulting from the temperature-dependent contributions of two domains with an easy magnetization axis along [100] and [010], respectively. Our study provides deep insights into the magnetic structure and its temperature dependence for this archetype magnetic film on the nanoscale.

\section{ACKNOWLEDGMENTS}

This work was performed at the Swiss Light Source, Paul Scherrer Institute, Villigen, Switzerland. The authors (C.E.VB, H.L.M., J.M.T., E.J., and M.P.) would like to thank the staff of the Swiss Light Source for their hospitality during our stay in Villigen. *hmeyerhm@ @mpi-halle.mpg.de
${ }^{1}$ M. Körling and J. Ergon, Phys. Rev. B 54, R8293 (1996).
${ }^{2}$ C. A. F. Vaz, J. A. C. Bland, and G. Lauhoff, Rep. Prog. Phys. 71, 056501 (2008). 
${ }^{3}$ J. Thomassen, F. May, B. Feldmann, M. Wuttig, and H. Ibach, Phys. Rev. Lett. 69, 3831 (1992).

${ }^{4}$ Dongqi Li, M. Freitag, J. Pearson, Z. Q. Qiu, and S. D. Bader, Phys. Rev. Lett. 72, 3112 (1994).

${ }^{5}$ R. E. Camley and D. Li, Phys. Rev. Lett. 84, 4709 (2000).

${ }^{6}$ T. Asada and S. Blügel, Phys. Rev. Lett. 79, 507 (1997).

${ }^{7}$ D. Spisák and J. Hafner, Phys. Rev. B 56, 2646 (1997).

${ }^{8}$ J. Miyawaki, A. Chainani, Y. Takata, M. Mulazzi, M. Oura, Y. Senba, H. Ohashi, and S. Shin, Phys. Rev. Lett. 104, 066407 (2010).

${ }^{9}$ D. Qian, X. F. Jin, J. Barthel, M. Klaua, and J. Kirschner, Phys. Rev. Lett. 87, 227204 (2001).

${ }^{10}$ H. L. Meyerheim, J.-M. Tonnerre, L. Sandratskii, H. C. N. Tolentino, M. Przybylski, Y. Gabi, F. Yildiz, X. L. Fu, E. Bontempi, S. Grenier, and J. Kirschner, Phys. Rev. Lett. 103, 267202 (2009).

${ }^{11}$ L. M. Sandratskii, Phys. Rev. B 81, 064417 (2010).

${ }^{12}$ H. Jenniches, J. Shen, Ch.V. Mohan, S. S. Manoharan, J. Barthel, P. Ohresser, M. Klaua, and J. Kirschner, Phys. Rev. B 59, 1196 (1999).

${ }^{13}$ M. Weinelt, S. Schwarz, H. Baier, S. Müller, L. Hammer, K. Heinz, and Th. Fauster, Phys. Rev. B 63, 205413 (2001).

${ }^{14}$ W. Keune, A. Schatz, R. D. Ellerbrock, A. Fuest, Katrin Wilmers, and R. A. Brand, J. Appl. Phys. 79, 4265 (1996).

${ }^{15}$ E. Kravtsov, D. Haskel, S. G. E. te Velthuis, J. S. Jiang, and B. J. Kirby, Phys. Rev. B 79, 134438 (2009).

${ }^{16}$ S. Roy, M. R. Fitzsimmons, S. Park, M. Dorn, O. Petracic, Igor V. Roshchin, Zhi-Pan Li, X. Batlle, R. Morales, A. Misra, X. Zhang, K. Chesnel, J. B. Kortright, S. K. Sinha, and Ivan K. Schuller, Phys. Rev. Lett. 95, 047201 (2005).

${ }^{17}$ J. M. Tonnerre, M. De Santis, S. Grenier, H. C. N. Tolentino, V. Langlais, E. Bontempi, M. Garcia-Fernandez, and U. Staub, Phys. Rev. Lett. 100, 157202 (2008).

${ }^{18}$ S. Brück, G. Schütz, E. Goering, X. Ji, and K. M. Krishnan, Phys. Rev. Lett. 101, 126402 (2008).

${ }^{19}$ U. Flechsig, F. Nolting, A. F. Rodriguez-Fraile, J. Krempasky, C. Quitmann, T. Schmidt, S. Spielmann, and D. Zimoch, AIP Conf. Proc. 1234, 319 (2010).
${ }^{20}$ U. Staub, V. Scagnoli, Y. Bodenthin, M. Garcia-Fernandez, R. Wetter, A. M. Mulders, H. Grimmer, and M. Horisberger, J. Synchrotron Radiat. 15, 469 (2008).

${ }^{21}$ J. P. Hill and D. F. McMorrow, Acta Crystallogr. Sect. A 52, 236 (1996).

${ }^{22}$ C. Kao, J. B. Hastings, E. D. Johnson, D. P. Siddons, G. C. Smith, and G. A. Prinz, Phys. Rev. Lett. 65, 373 (1990).

${ }^{23}$ C. C. Kao et al., Phys. Rev. B 50, 9599 (1994).

${ }^{24}$ J. M. Tonnerre, L. Seve, D. Raoux, G. Soullie, B. Rodmacq, and P. Wolfers, Phys. Rev. Lett. 75, 740 (1995).

${ }^{25}$ H. L. Meyerheim, R. Popescu, D. Sander, J. Kirschner, O. Robach, and S. Ferrer, Phys. Rev. B 71, 035409 (2005).

${ }^{26}$ J. Zak, E. R. Moog, C. Liu, and S. D. Bader, Phys. Rev. B 43, 6423 (1991).

${ }^{27}$ Z. Q. Qiu and S. D. Bader, Phys. Rev. Sci. Instrum. 71, 1243 (2000).

${ }^{28}$ C. T. Chen, Y. U. Idzerda, H. J. Lin, N. V. Smith, G. Meigs, E. Chaban, G. H. Ho, E. Pellegrin, and F. Sette, Phys. Rev. Lett. 75, 152 (1995).

${ }^{29}$ MOKE experiments have indicated, after applying the magnetic field normal to the film surface, the presence of out-of-plane hysteresis. This effect is compatible with the magnetic structure model with a combination of stable and variable features. In this study, we focus on the robust aspects: the presence of the spin blocks in the magnetic structure and inhomogeneity of the temperature dependence of magnetization within the film. The systematics and statistics of the variable features sensitive to the sample fabrication and sample history need extensive additional efforts and are outside of the scope of this paper.

${ }^{30}$ The analysis includes the independent transverse angle $\gamma$ attributed to each spin block, namely, $[\uparrow \uparrow \downarrow],[\uparrow \downarrow]$, and $[\uparrow]$, corresponding to the layers [Nos. 4-6], [Nos. 2-3], and [No. 1], respectively. Within the experimental uncertainty of about $5^{\circ}-15^{\circ}$ on average, the magnetization of all blocks are collinear. The collinearity of the in-plane component of the magnetization is coherent with the existence of a magnetic anisotropy in the sample plane.

${ }^{31}$ V. Scagnoli, U. Staub, Y. Bodenthin, M. Garcia-Fernandez, A. M. Mulders, G. I. Meijer, and G. Hammerl, Phys. Rev. B 77, 115138 (2008). 